FORMATION Formation emploi

Revue française de sciences sociales

99 | juillet-septembre 2007

Les usages sociaux de la compétence

\title{
La fabrication des compétences, un processus piloté par l'aval?
}

\section{Marcelle Stroobants}

\section{(2) OpenEdition}

1 Journals

\section{Édition électronique}

URL : http://journals.openedition.org/formationemploi/1491

DOI : 10.4000/formationemploi.1491

ISSN : 2107-0946

\section{Éditeur}

La Documentation française

\section{Édition imprimée}

Date de publication : 1 juillet 2007

Pagination : 89-94

ISSN : 0759-6340

\section{Référence électronique}

Marcelle Stroobants, «La fabrication des compétences, un processus piloté par l'aval ? », Formation emploi [En ligne], 99 | juillet-septembre 2007, mis en ligne le 19 février 2009, consulté le 30 octobre 2020. URL : http://journals.openedition.org/formationemploi/1491 ; DOI : https://doi.org/10.4000/ formationemploi.1491 


\section{POSTFACE}

\section{La fabrication des compétences, un processus piloté par l'aval ?}

Par Marcelle Stroobants*

Cela fait presque vingt ans maintenant que la compétence règne dans les milieux du travail et de la formation, sans rien avoir perdu de son ambiguïté ni de sa polysémie.

Dans son introduction, Sylvie Monchatre rappelle fort justement que la sociologie cherche à comprendre les enjeux de cette construction socialisée à partir de ses effets, ce qu'elle appelle «l'aval du processus». En même temps, elle amorce un détour par l'amont, l'étymologie et le sens traditionnel du terme compétence selon le dictionnaire. Cette préoccupation nous semble aussi significative que féconde dès lors qu'il est question de construction sociale.

\section{QUELLES CONSTRUCTIONS SOCIALES ?}

Affirmer qu'un phénomène est une construction sociale apparaît souvent comme une mise en garde réductrice, reçue comme un «ne que», comme si le phénomène en question, faute d'être naturalisable, ne pouvait donc être $q u$ 'une vue de l'esprit, sans consistance ni efficacité, vouée à rejoindre le magasin des artifices. Sous cet angle, les compétences ne seraient que des appellations consacrées, que des conventions, des étiquettes arbitraires, comme on a pu l'affirmer, au demeurant, à propos des qualifications. L'exemple des catégories de genre devrait permettre de lever aussitôt cette réduction. La fameuse formule «on ne naît pas femme on le devient » incite à penser le genre en construction, sans rien lui retirer de sa puissance. Cette conception n'implique pas, en effet, qu'on puisse moduler à sa guise ce devenir, l'apprivoiser ou se débarrasser aisément de tout ce qu'il nous a fabriqué dans la foulée, à commencer par des (in)compétences. C'est tout un programme de recherche qui s'ouvre à partir de là pour comprendre les ressorts de ce devenir et donc aussi le processus lui-même.

Pour surmonter l'alternative entre la naturalisation des compétences et leur déni, le dictionnaire le plus ordinaire rouvre une perspective intéressante, puisque la définition de compétence y comporte toujours au moins une double connotation, à la fois juridique et cognitive, ou si l'on préfère, conventionnelle et effective. La compétence serait en même temps la capacité et sa reconnaissance, l'aptitude et le droit de l'exercer. Cette conjonction répond à notre pari de voir

* Marcelle Stroobants est sociologue. Elle enseigne la sociologie du travail à l'université Libre de Bruxelles. Ses travaux portent sur le travail, la qualification, la compétence. Elle a notamment publié : Sociologie du Travail, Armand Colin, collection « 128 », Paris, nouvelle édition 2007. "L'individualisation des relations salariales par la gestion des compétences ", in Dupray A., Guitton C., Monchatre S. (Coordination), Réfléchir la compétence. Approches sociologiques, juridiques, économiques d'une pratique gestionnaire, Toulouse, Octares, 2003, pp. 43-52. 
la compétence comme le produit d'un processus d'habilitation qui littéralement habilite à acquérir et exercer un certain type d'habileté. Ce processus est envisagé comme une transformation complexe, sociale, une mise en forme dont l'issue n'est pas déterminée'. Autrement dit, la compétence n'a d'existence que si elle est jugée comme telle, ce qui n'exclut pas l'éventualité de deux autres cas de figure inachevés, celui de la reconnaissance présomptueuse et celui de la capacité méconnue. Le regard sociologique est donc aussi amené à se porter en amont, non seulement vers les institutions de formation, mais aussi vers les épisodes au cours desquels des compétences s'acquièrent.

La compétence est socialement reconstruite en un second sens, en tant que notion produite par les interactions entre constructions «savantes» et «profanes» sur le terrain du travail, pendant les années 80 . En suivant ces mouvements de l'intérieur, en cours de production, en amont de son succès, on découvre l'ampleur de notre ignorance en matière d'apprentissage, de transfert et même d'identification des compétences.

\section{REGARD RÉTROSPECTIF EN AMONT}

Parmi les principaux protagonistes de la fabrication des compétences, il y a évidemment les responsables des stratégies de gestion dans les entreprises. À y regarder de plus près, on peut constater cependant que deux autres types d'ingrédients y jouent un rôle décisif, les technologies et les chercheurs. Parmi ces derniers, certains participaient à des programmes de recherche de type «technologie, emploi, travail», d'autres poursuivaient des controverses antérieures. Les divisions du travail entre disciplines ou entre praticiens et théoriciens semblent alors moins pertinentes pour les départager que les points de vue qu'ils adoptent.

\footnotetext{
${ }^{1}$ Au-delà d'une relation interpersonnelle, l'habilitation désigne, pour nous, une configuration susceptible de favoriser, enregistrer et entériner la résolution d'un problème au sens de la « transduction » chez Simondon. Dans ce cas, la transformation passe par l'invention d'une association entre des forces internes et une exigence extérieure (Stroobants, 1993). Les exemples considérés à l'époque rappellent aussi ces plug-ins, ces sortes de technologies intellectuelles distribuées, évoquées par Bruno Latour (Latour, 2006).
}

\section{Les chercheurs}

Sous l'angle de la sociologie du travail'2, les raisonnements qui ont alimenté le grand mouvement des compétences n'apportent guère de renouvellement théorique. Ces raisonnements reproduisent, en effet, une conception naturalisante de la qualification qu'on aurait pu penser récusée, en considérant notamment toutes les études sur le travail des femmes. Ces études ont entre-temps pu confirmer à quel point les qualifications résultaient de rapports de force et non des caractéristiques intrinsèques des tâches. Néanmoins, l'argument selon lequel les qualités du travail seraient directement observables et plus réelles que les formes qui les structurent n'en revient pas moins à la charge. Voyons de quelle manière.

Dès le milieu des années 80 s'ébauchent notamment deux courants qui annoncent, chacun à leur façon, l'importance des compétences.

Un premier courant s'inscrit en réaction aux travaux antérieurs qui avaient pu dénoncer la dégradation du travail et des qualifications. Toute une série d'observations fines, souvent assistées par des ergonomes, attestent alors que les travailleurs déploient des stratégies insoupçonnées, des formes de créativité et d'initiative, préalablement négligées. Dans cette perspective, les travailleurs s'avèrent plus compétents qu'on ne le croyait. Cette réfutation s'adresse aux chercheurs qui avaient pu sous-estimer la richesse des interventions informelles, mais aussi aux rationalisateurs et autres concepteurs de machines. De là à dire que les qualifications « conventionnelles » ne rendent pas justice à tous ces savoir-faire, à toutes ces compétences «réelles», il n'y a qu'un pas, que certains auteurs franchissent aussi allégrement que plusieurs de leurs prédécesseurs.

Un second courant de recherche tend plutôt à soutenir que des compétences différentes et plus

\footnotetext{
${ }^{2}$ La sociologie du travail a beaucoup tardé à s'intéresser à ce phénomène, alors même que certains de ses représentants ont pu participer - positivement ou indirectement - à la propagation et au durcissement de la notion de compétence. Il a fallu attendre 2001 pour que la revue Sociologie du travail y consacre un numéro spécial. Entre-temps, deux épisodes importants ont pu contribuer à éveiller l'intérêt des théoriciens du travail à cet égard, les Journées d'études organisées par le MEDEF (Mouvement des entreprises de France) en 1998 et l'essai de Boltanski et Chiapello en 1999.
} 
importantes qu'avant seraient mobilisées - en fait requises - au travail. Ici l'accent est surtout mis sur des capacités de communication, des qualités de gestion, des savoir-être, voire des «qualifications sociales ». Ce courant-ci se place moins dans une perspective de réfutation que dans celle d'une révélation, tantôt proclamée sur le mode indicatif - les choses ont changé - tantôt sur le mode prescriptif - les choses doivent changer -.

Cette lecture tend donc à relativiser l'originalité des deux raisonnements aussi bien que les arguments empiriques avancés. Ces deux courants apparaissent bien comme des résurgences de la conception substantialiste de la qualification au sens où ils entendent éprouver les qualifications à partir des qualités du travail. L'un et l'autre participent potentiellement au phénomène qu'il s'agirait d'étudier, le jugement social sur ce qui est digne d'être qualifié. Le premier courant s'appuie sur les qualités supposées acquises par les travailleurs et le second sur les nouvelles qualités supposées requises par le travail. Tous deux adoptent un point de vue susceptible d'apporter des arguments aux parties impliquées dans la négociation de la qualification, le premier aux travailleurs, le second aux employeurs. Faute de controverse susceptible d'épuiser ces partis antagoniques, le second courant finira par l'emporter.

Cette lecture confirme aussi l'impossibilité de trouver dans le travail un critère objectif pour en justifier la valeur relative. Car il n'existe manifestement aucune unanimité sur la définition de ces compétences qui seraient plus importantes que prévu ou plus importantes qu'auparavant. La seule constante observable relève de la catégorisation plutôt que du contenu. À la fin de cette décennie, un certain consensus semble établi sur le fait que les compétences désignent la capacité à mobiliser des savoirs, des savoir-faire, des savoir-être. Cette fameuse trilogie qu'on retrouve aussi dans la sphère éducative s'est elle-même élaborée à coup de redressements progressifs. « Non seulement» le travail mobilise des savoirs, «mais aussi» des savoir-faire et «non seulement» des savoir-faire, «mais encore» des savoirs non techniques, sociaux ou des savoir-être. Chacun de ces «non seulement» fonctionne un peu comme un repoussoir de l'évidence. « Non seulement des savoirs » laisse entendre qu'il y a plus dans la pratique professionnelle que ces évidentes connaissances formelles, théoriques, transmises par la formation initiale. «Non seulement des savoir-faire» suggère qu'au-delà des procédures et des opérations techniques, il y a tant d'indicibles comportements, des manières d'être, du social donc, insaisissable, plus informel que tout autre. Si la compétence mobilise des savoirs plus sociaux que la qualification, que devient une «compétence sociale»? Une qualification sociale au carré ? Mais en fait, que serait une qualification qui ne soit pas sociale? On conviendra - non sans interrogation - que cette trilogie confuse peut s'appliquer à tout, y compris au passé. Comment comprendre la soudaineté de cette découverte?

\section{Les technologies}

À la fin des années 80 , le ton se précise, le second courant est devenu dominant, non sans avoir intégré certains arguments du premier. À ce moment-là, la mobilisation des ressources humaines apparaît comme une pièce maîtresse du discours sur la mutation du système productif, propagé par la littérature managériale. Dans cette perspective, la mutation associée aux nouvelles technologies exigerait des compétences d'un nouveau type, échappant aux anciennes formalisations et les condamnant du même coup. Il s'agit bien d'une offensive dont la cible se précise encore, la qualification et, avec elle, le diplôme. Les seuls facteurs de mutation ici invoqués, les TIC (technologies de l'information et de la communication), n'en exercent pas moins un rôle déterminant. En serait-on revenu à une perspective évolutionniste, caricaturant en quelque sorte les trois phases du travail envisagées par Touraine au milieu des années 50 ? On se souvient que dans la phase $\mathrm{C}$, celle de l'automatisation de la fabrication, la qualification de l'ouvrier n'était plus directement reliée aux instruments de production. Mais la «qualification sociale » selon Touraine définissait des capacités de communication, avec un statut reconnu. Peu après, lorsque Naville réfléchissait au cas des industries précocement automatisées, ce n'était pas pour tenter de justifier la qualification à partir de la nature du travail, mais exactement le contraire. Quand la production s'automatise, quand l'organisation du travail s'émancipe d'une division technique, d'autres critères peuvent être utilisés pour hiérarchiser les 
valeurs attribuées aux travaux. La société n'a finalement pas trouvé de meilleure convention pour statuer sur la complexité d'un travail que de l'appréhender par la durée de formation de celui qui l'exerce, le niveau du diplôme servant d'indicateur de formation initiale, l'ancienneté servant d'indicateur d'expérience.

En ce sens, on pourrait dire que les technologies d'automatisation déterminent une indétermination, une plus grande marge de manœuvre dans la construction des qualifications.

Or, trente ans plus tard, lorsque l'informatisation étend l'automatisation dans l'industrie et les services, ces repères traditionnels de la qualification sont brusquement déclarés périmés. Les compétences semblent n'avoir aucun rapport avec les acquis scolaires, comme si elles étaient toujours déjà là, potentiellement en germe, ou comme si elles naissaient de la situation où elles sont mobilisées. Et pourtant, certaines - et non des moindres - sont censées être transférables. Elles doivent être bien spectaculaires pour susciter tant d'émois et de remous. Or précisément pas. Ces compétences ne se voient pas, sont informelles et leur existence ne peut qu'être présumée à partir d'une performance particulière.

Comment parler de révélation si les nouvelles compétences sont invisibles ?

Si les TIC ont pu jouer un rôle dans le succès de la notion de compétence, c'est précisément celui d'un révélateur. Car toutes ces sortes de savoir-faire, de savoirs tacites, de talents insoupçonnés apparaissent manifestement comme un attribut humain par contraste avec les tentatives de les simuler artificiellement. L'intelligence des travailleurs, c'est d'abord ce qui dépasse les promesses de l'intelligence artificielle. Mais ce qui les différencie radicalement des machines ne suffit pas forcément à les qualifier les uns par rapport aux autres. D'autre part, pour piloter ou surveiller la production assistée par ordinateur, il faut savoir faire autre chose qu'avant. Toutes les qualités supposées requises se voient alors déclinées dans le vocabulaire précisément utilisé pour caractériser ces technologies cognitives, de l'information et de la communication. En ce sens, c'est autant la manière de percevoir les technologies elles-mêmes - et les surprises qu'elles ménagent - qui a pu servir de révélateur. Des compétences invisibles sont envisagées avant tout parce qu'on a changé de manière de voir. Or ce travail « réel » là n'est pas moins représenté, n'est pas moins mis en forme, que tout autre. La promotion des compétences ne semble donc pas non plus répondre à une nécessité empirique.

\section{EN AVAL, LES PRATIQUES}

Que peut donc apporter cette notion qui ne répond à aucune exigence théorique ni empirique?

Au tournant des années 90, tandis qu'est mise en doute la possibilité de prescrire, former, évaluer, ou tout simplement définir les compétences, des tentatives en ce sens sont déjà à l'œuvre, dans les réformes éducatives et dans les entreprises (Ropé et Tanguy, 1994).

Ici, des référentiels de formation tentent de redéfinir des objectifs à atteindre, abstraction faite des connaissances à transmettre. Là, des bilans de compétence font miroiter la possibilité de reconnaître des acquis méconnus, mais la validation de l'expérience ne peut se départir de la référence aux certifications scolaires. Des parcours de mobilité sont cartographiés en spéculant sur des affinités cognitives déconnectées des traditions du marché de l'emploi ${ }^{3}$. D'un côté, on spécule sur des compétences plus transversales qu'un métier, d'un autre on admet qu'une compétence ne s'évalue que dans une action particulière. Et quoi de plus transférable que les compétences signalées par un diplôme?

Tous ces paradoxes découlent du postulat mythique d'accès direct à une «réalité » de l'activité, plus

\footnotetext{
${ }^{3}$ Les aires de mobilité du ROME, Répertoire opérationnel des métiers et des emplois, éditées par l'ANPE dès 1993, sur base de proximités obtenues par inférences, en constituent l'illustration. Le résultat le plus fascinant de ce traitement est certainement l'invention des compétences présumées transférables entre un employé des pompes funèbres, un pédicure-podologue, un déménageur ou un testeur sensoriel, par exemple. On devine la surprise de l'entrepreneur de pompes funèbres - et celle de Julien Bernard dont l'article ici même souligne l'importance de la gestion des émotions dans ce métier - face à un candidat au recrutement qui s'affirmerait compétent comme opérateur funéraire puisqu'il a été ouvrier du béton.
} 
réelle, pense-t-on, que les représentations qui prévalaient jusqu'alors. Les tentatives de formaliser de l'informel, abstraction faite des codifications antérieures, vont d'ailleurs rencontrer exactement le même problème que celui posé par la rédaction d'un questionnaire totalement dénué d'ambiguïté ou d'un mode d'emploi universel. Le souci d'être compréhensible par un lecteur générique s'épuise dans la généralisation incompréhensible ou le retour d'une infinité de spécifications particulières ${ }^{4}$.

C'est encore cette même fiction du travail réel qui est à l'œuvre dans la stratégie de gestion des compétences. L'emploi du même terme «compétence» pour caractériser les capacités requises par les emplois et les capacités supposées acquises par les travailleurs semble à première vue permettre de faire l'économie des opérations qui président à leur identification et à leur articulation ${ }^{5}$. L'équivalence semble directe et automatique. Cette apparente simplification de la relation entre formation et emploi tend à évincer tous les jugements qui interviennent de part et d'autre, les compromis pratiqués sous le règne de la qualification, voire à court-circuiter les conventions externes et collectives. En fait, l'équivalence simplifiée recouvre tout un travail d'élaboration de dispositifs locaux d'évaluation et le souci de rupture avec les anciennes codifications s'avère finalement démesuré .

Depuis sa signature en 1990, l'accord Cap 2000, emblème de la logique compétence dans la sidérurgie, a été abondamment commenté, tour à tour envisagé comme une innovation nécessaire et prometteuse ou comme une réforme mitigée plus ou moins menaçante.

\footnotetext{
${ }^{4}$ Cette sorte «d'abîme de l'exécution » (Latour, 2006, p. 301) rencontré lors de l'élaboration des National Vocational Qualifications, au Royaume-Uni, n'a d'ailleurs été surmonté qu'en explicitant les éléments de compétences par des références contextuelles familières (Wolf, 1994).

${ }_{5}$ «La compétence est cette unité de conversion qui permet de comparer et de mettre en équivalence, comme le fait la monnaie sur le marché, des "besoins" définis par les organisations dans l'entreprise et des "ressources humaines" " (Baron, 1993, p. 7).

${ }^{6}$ "Il faut bien se rendre à l'évidence de l'absence de modèle crédible et réaliste susceptible de prendre le relais de celui légué par M. Taylor. Il est aujourd'hui facile de faire le bilan des effets pervers du taylorisme. Il est beaucoup moins simple de le remplacer » (Baron, 1993, p. 18).
}

Contrairement aux intentions annoncées, l'évaluation des compétences requises ne remet pas forcément en question la division du travail. Tout un travail de décomposition-recomposition en familles de métier, dans la continuité des évaluations de fonctions, a pu aboutir à redéfinir des emplois-types à géométrie variable. La logique du poste reste donc d'application, mais elle est plus flexible.

D'autre part, l'évaluation des compétences acquises et leur mise en rapport avec les emplois brigués passent dans ce «modèle» par un entretien individualisé. Le diplôme, décrété encombrant, reste une référence incontournable sur le terrain; en revanche, la règle de progression à l'ancienneté est abolie.

Cette orientation a pu paraître s'inscrire dans la continuité des revendications des ouvriers métallurgistes des années 70 , plaidant pour la reconnaissance de leur professionnalité effective. Cette stratégie syndicale classique représentait, en fait, une demande de reconnaissance par catégorie de travailleurs, qui n'a pas grand-chose à voir avec une évaluation des capacités individuelles.

Au-delà de la sidérurgie, les méthodes de classification sophistiquées deviennent d'autant plus difficiles à contrôler collectivement que leur conception est commandée à des consultants privés qui n'ont aucun intérêt à divulguer leurs secrets de fabrication. La détermination de salaires équitables n'apparaît plus ainsi comme une affaire de négociations, mais comme une opération technique, à confier à des experts. Les accords signés témoignent d'une redéfinition des rôles de la branche et de l'entreprise, dont l'effet dépend néanmoins de la dynamique des relations collectives. La régulation de branche tend à se focaliser sur des procédures dont la mise en œuvre reste dévolue à l'entreprise.

La logique compétence vise généralement à remplacer les règles impersonnelles de progression à l'ancienneté et de rétribution suivant le poste par la progression et le salaire individualisés. Le postulat de transparence du travail «réel » conduit à des normes simplificatrices, ou à des prescriptions abstraites et hermétiques. Les salariés et leurs représentants ne peuvent qu'éventuellement contester les résultats de l'évaluation après coup. Les syndicats, écartés du contrôle des évaluations, demeurent peu enclins à intervenir dans la 
gestion, prérogative de l'employeur, sous peine de s'exposer au clientélisme (Zimmermann, 2000).

En définitive, l'obligation de résultat associée à la mise en compétition par l'évaluation personnelle concourent à faire de la compétence un instrument de flexibilité et de gestion par le stress.

L'émergence de la notion de compétence, à la fin des années 80 , aurait pu passer pour un changement de vocabulaire, dans un souci de modernisation de la qualification, souvent marquée par des connotations ouvriéristes. En amont de cette construction, l'opération s'avère plus radicale puisqu'elle passe par la mise à l'épreuve du régime associé à la qualification. La qualification se signalait par des attributs, une durée de formation, une appellation contrôlée collectivement, un label de qualité. La compétence apparemment plus réelle, plus naturelle, se décline sur le mode de l'être. Incorporée à la personne, elle s'apparenterait à du «capital humain », à ceci près que sa formation et son évaluation emprunteraient des voies très incertaines.

Seule une pratique gestionnaire ne peut suffire à supplanter l'ensemble des régulations salariales. Mais cette pratique ne constitue pas, on l'a vu, la seule application de la notion de compétence. Dans une économie dite cognitive, la formation des compétences n'apparaît plus désormais comme une obligation collective, mais comme un investissement individuel et perpétuel. Car pour les employeurs qui font appel à une main-d'œuvre qualifiée, explique Annie Vinokur (Vinokur, 2005), "le problème est donc de piloter souplement "par l'aval" et à flux tendu une production des compétences dont ils n'aient pas à supporter le coût ».

\section{Bibliographie}

Baron X. (1999), «La gestion prévisionnelle des emplois et des compétences en entreprise », Cahiers Français, 262, pp. 3-18.

Boltanski L. et Chiapello E. (1993), Le nouvel esprit du capitalisme, Paris, Gallimard.

Dupray A., Guitton C. et Monchatre S. (2003), Réfléchir la compétence. Approches sociologiques, juridiques, économiques d'une pratique gestionnaire, Octarès.

Latour B.(2006), Changer de société. Refaire de la sociologie, Paris, La Découverte.

Ropé F., Tanguy L. (Éd.) (1994), Savoirs et compétences. De l'usage de ces notions dans l'école et l'entreprise, Paris, L'Harmattan.
Stroobants M. (1993), Savoir-faire et compétences au travail au travail. Une sociologie de la fabrication des aptitudes, Bruxelles, Éditions de l'université de Bruxelles.

Vinokur A. (2005) «Qui pilote l'éducation?», in Nouveaux Regards, $\mathrm{n}^{\circ}$ 29, avril-juin, pp. 11-15.

Wolf A. (1994), «La mesure des compétences: l'expérience du Royaume-Uni », Revue européenneFormation professionnelle, 1, pp. 31-38.

Zimmermann B. (2000), «Logiques de compétences et dialogue social», Travail et Emploi, $\mathrm{n}^{\circ} 84$, octobre. 